\title{
Reversing coronary artery calcium using a functional medicine protocol
}

\begin{abstract}
Introduction: Coronary artery disease (CAD) is the leading cause of early mortality in Western countries. Coronary artery calcium (CAC) is a reliable study to predict future myocardial events and offers independent cardiac risk information. Standard CAD medications, statins, do not reverse CAC. CAD is a chronic disease mainly caused by chronic inflammation. Reversing or partially reversing CAC using a functional approach, without any prescription medications, is theoretically possible due to the chronic inflammatory nature of CAD.
\end{abstract}

Diagnosis: Diagnosis and follow-up of CAC can be easily performed with CT imaging.

Interventions: A baseline cardiac scan was performed, and the presence of mild CAC was confirmed in a 60-year-old male. A functional approach, without any prescription medications, was started a few months later and continued for 12 months. A repeat CT scan performed at this time, showed a significant CAC improvement.

Conclusion: A twelve-month functional approach, without prescription medications, successfully decreased CAC

Keywords: coronary artery disease, coronary artery calcium, reversing coronary artery calcium
Volume I4 Issue 2 - 202 I

\section{Manuel Moran}

Department of Surgery,Veterans Administration Medical Center, USA

\author{
Correspondence: Manuel Moran, 480 IVeterans Drive, \\ Veterans Administration Medical Center, USA, Saint Cloud, MN \\ 56303, USA, Tel I-320-255-6429, Fax 1-320-255-6406,
} Email manuel.moran@va.gov

Received: April 03, 2021 | Published: April 22, 202
Abbreviations: CAD, coronary artery disease; CAC, coronary artery calcium; HIIT, high intensity interval exercise; BMI, body mass index

\section{Introduction}

Coronary artery disease (CAD) is a chronic inflammatory condition By now, it is well proven that saturated animal fats do not because $\mathrm{CAD}$, neither does dietary cholesterol. The main etiology is chronic inflammation produced by processed foods. All man-made fats and processed oils, as well as all refined carbohydrates, are responsible for this disease. Offending fats include all processed vegetable oils, as well as trans fats (hydrogenated or partially hydrogenated oils) and interesterified fats. Before processed foods became available, CAD did not exist. For example, mortality data from Boston showed no cardiac disease deaths in the year 1811. Other contributing factors for CAD include nutritional deficiencies and toxins like smoking cigarettes. Heavy metals, like lead, increase CAD. Common nutritional deficiencies that contribute to CAD include vitamin $\mathrm{K} 2$, intracellular magnesium, and vitamin D3. The working hypothesis was to decrease CAC using a multifaceted functional approach by: (A) eliminating well known etiological factors; (B) replacing any possible nutritional deficiencies; and $(\mathrm{C})$ implementing a detoxifying protocol.

CAC is a very good test to screen for CAD in asymptomatic patients. A multivariable analysis in the MESA study showed that the only predictive variable was a coronary artery scan score greater than $100 .^{1}$

\section{Material and methods}

60 -year old asymptomatic male was found to have mild CAC on a screening coronary scan, mostly affecting the left coronary artery. No prescription medications. No significant past medical history, never smoked. Patient did not have any risk factors except for a history of large refined carbohydrate intake, work-related stress, and a history of processed food consumption. All standard laboratory tests were normal, including lipid panel, high-sensitive (cardiac) C-reactive protein, fasting insulin, homocysteine, serum ferritin, fibrinogen, apolipoprotein B, vitamin D3 level, and A1c hemoglobin (mildly elevated at 5.7 two years prior but with quick normalization after instituting dietary changes). BMI was within normal limits at 19 (weight $=56 \mathrm{~kg}$ ). Dangerous heavy metal levels and other toxic products were ruled out with a hair tissue mineral analysis.

A functional protocol was followed for 12 months and consisted of:

I. Diet, mostly plant-based, organic, without any caloric restrictions

A. Organic whole foods, raw or cooked at low temperature: Fat consumption was not restricted, thus it was a normal or high-fat diet, normal or low protein. This diet included a large amount of vegetables, many nuts (except for cashews, no peanuts which are legumes), garlic and black garlic (both garlic decrease arterial plaque), and many leafy vegetables. No restrictions on whole fruits but no fruit juices. Sauerkraut which is a probiotic. Green or hibiscus tea brewed with reverse osmosis water, which was the only drinking water used (free of fluoride and chlorine). Hibiscus tea at $10 \mathrm{~g} / \mathrm{cup}$ daily or twice daily decreases CAD. No processed foods. Thus, no processed vegetable oils or any other man-made fats. No fast-food restaurant meals. No canned or frozen foods. No artificial sweeteners. Monk fruit, stevia, and allulose were used as sweeteners. No sodas.

B. No refined carbohydrates.

C. 1.4. No grains. Thus no wheat or any type of gluten because it increases inflammation by several mechanisms, including a leaky gut. 
D. 1.5. No dairy products, which are inflammatory. 1.6. 10-15\% of animal products (free range eggs, grass-fed beef, etc.) Animal products were minimized because $90 \%$ of toxins ingested are from this source, compared to $10 \%$ from plantbased foods.

E. Quarterly, five-day fast $(<400 \mathrm{cal} /$ day $)$ or fast-mimicking diet as described by Dr. Valter Longo.

F. Exercise. HIIT 2-3 times weekly. It increases the number of mitochondria and improves insulin sensitivity.

G. Stress control. Meditation at least 2-3times weekly. Chronic stress is a major contributor to CAD.

H. Supplements.

a. Vitamin K2, MK-7 600mcg daily.

b. Vitamin K2, MK-4 15,000mcg three times daily. This dosage is used in Japanese clinical studies to treat osteoporosis.

c. Vitamin D3 5,000 IU daily. Vitamin D3 levels should be on the highest range of normal.

d. Magnesium oxide 400mg daily. Dissolves calcium deposits.

e. Magnesium chloride $1,000 \mathrm{mg}$ daily (has $120 \mathrm{mg}$ of magnesium). Prevents ectopic calcium deposits.

f. Magnesium citrate $400-500 \mathrm{mg}$ daily g. High quality, mercury free, fish liver or cod liver oils (no capsules), $5-10 \mathrm{ml}$ per day.

h. Multivitamins and minerals

i. Vitamin C 1,000mg supplement in addition to daily lemon juice (one lime or lemon per day)

j. Stone root 900mg twice daily. Dissolves calcium deposits.

$\mathrm{k}$. Chanca piedra 1,000mg three times daily. Also dissolves calcium deposits.

1. Ubiquinol 100mg daily. Improves energy production.

$\mathrm{m}$. No calcium supplements because they increase CAC.

\section{Detoxification}

a. Infrared sauna, 25-30minutes at 54-65 degrees Celsius (130-150 degrees Fahrenheit) 2-4 times weekly for 20-30 minutes. Toxins are excreted in the sweat.

b. Liquid EDTA (Cardio Renew $\left.{ }^{\circledR}\right) 14$ drops in $50 \mathrm{ml}$ of water five times daily $\mathrm{x} 5$ weeks, then once daily for maintenance. Liquid EDTA is better absorbed than other oral forms. (Note: liposomal EDTA is available but much more expensive). EDTA binds and eliminates heavy metals but does not decrease CAC.
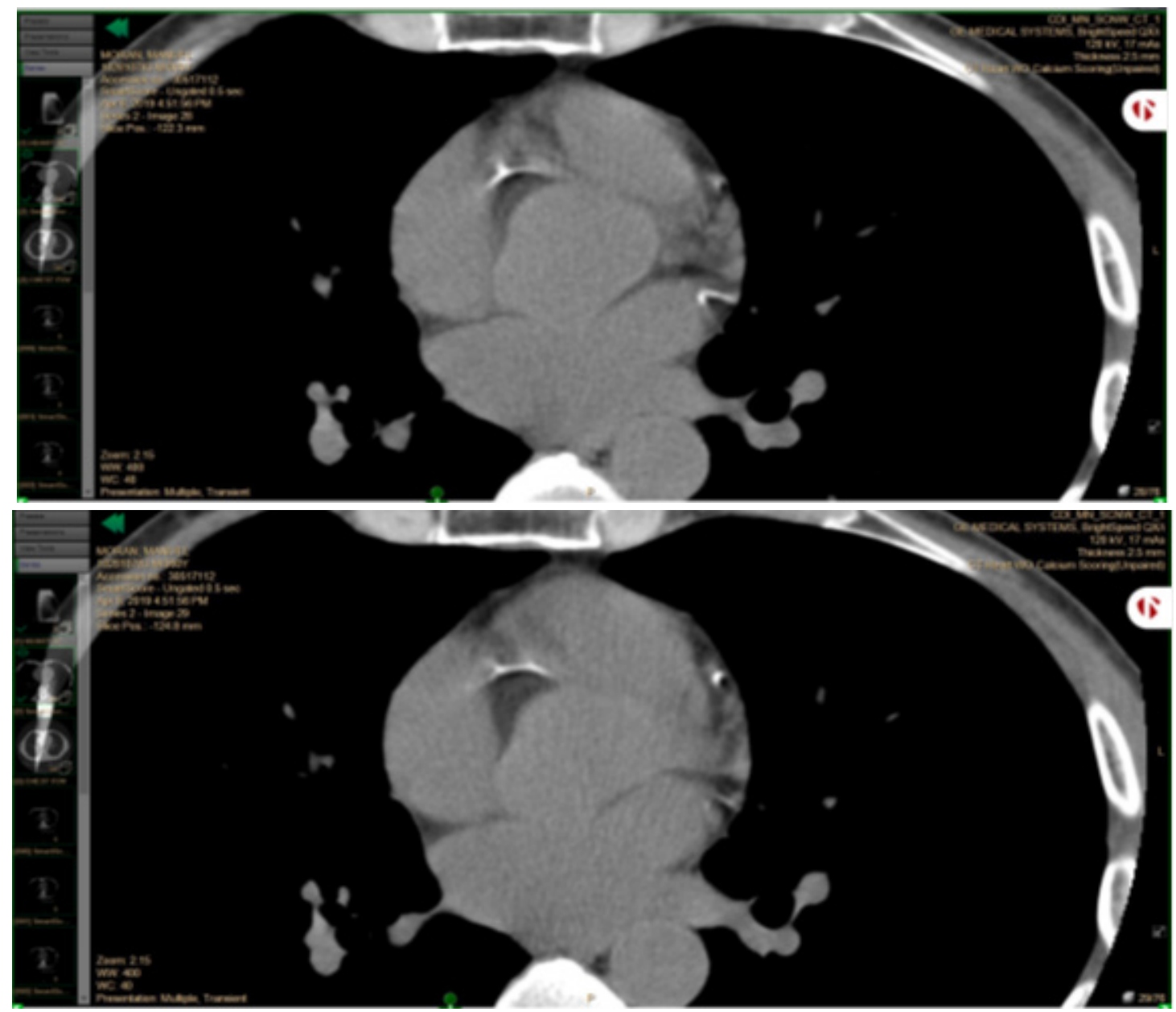

Citation: Moran M. Reversing coronary artery calcium using a functional medicine protocol.J Cardiol Curr Res. 202I; I4(2):38-4I. 

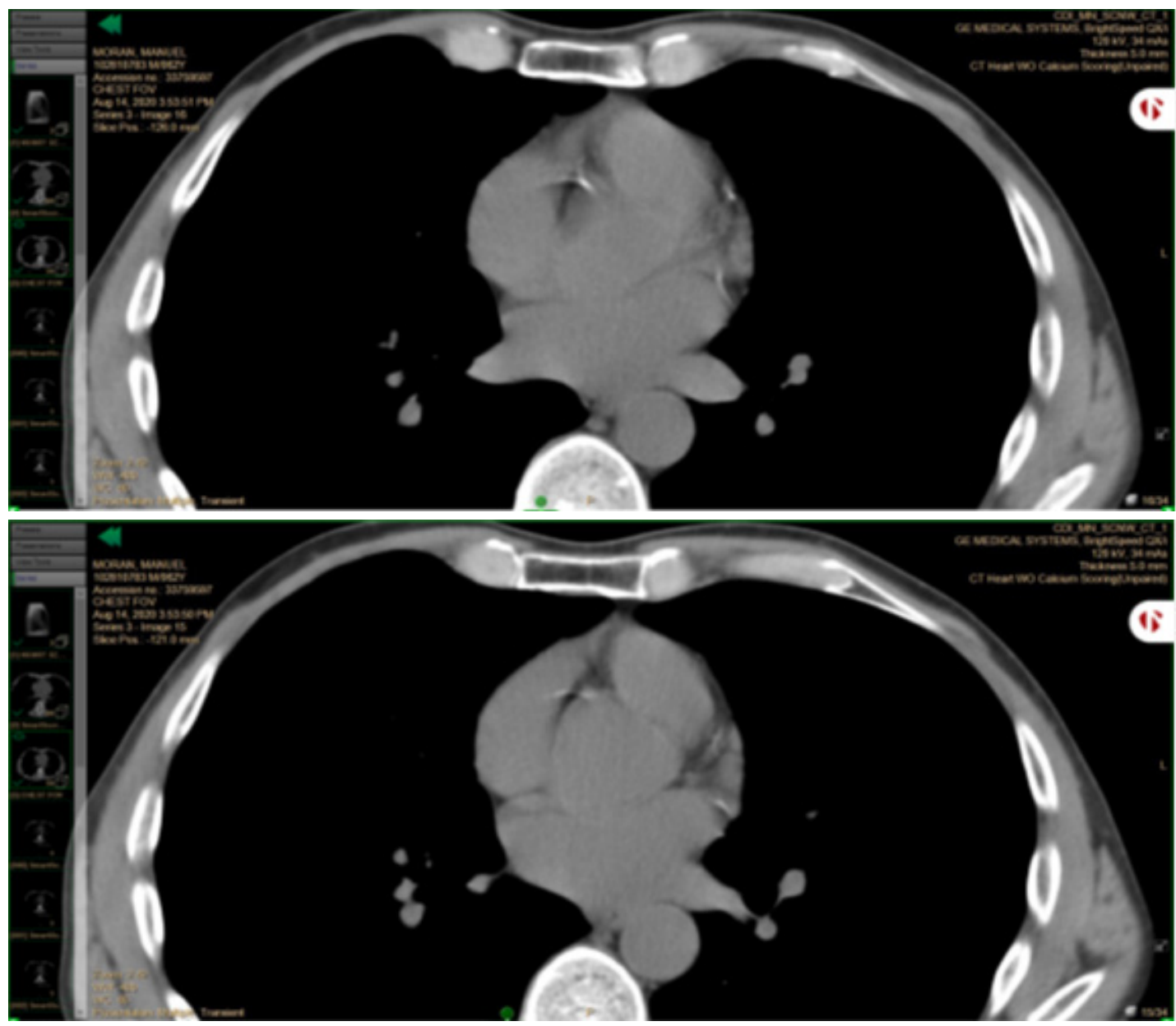

\section{Results}

No cardiac events happened during the study period. The right coronary artery calcium score dropped from 39 in 2019 to 21 in 2020. Circumflex calcium score dropped from 9 to 2. In the CT images below, left upper cardiac artery is the right coronary artery; right lower artery is the circumflex.

\section{Discussion}

High-fat diets do not cause CAD if healthy fats are consumed. Well known tribes, like the Atoll people in the Tokelau Island and the Maasai tribe in Kenya and Tanzania, consume a large amount of animal fat (66\% in the Maasai diet) or large amount of saturated fats (Atoll people eat $54-62 \%$ of their caloric intake from coconut oil) but do not suffer from CAD. Many studies in Western countries have found no correlation between cholesterol intake and CAD Some famous CAD reversing protocols have focused on very lowfat diets, like the Ornish and the Esselstyn diets, but have had very limited success reverting CAD although were successful decreasing new cardiac events. Nevertheless, most people will not be willing to stay on a low or very low-fat diet forever. Because healthy fats do not cause $\mathrm{CAC}$, it makes no sense to promote very low-fat diets to prevent or reverse $\mathrm{CAC}$ or $\mathrm{CAD}$.

Once CAC is detected, reversal with statins has not been successful. In fact, CAC progression continues. CAC score typically worsens $20-25 \%$ per year. Dr. William Davis (cardiologist) thinks a $5 \%$ increase per year is a successful therapy. 615 postmenopausal women underwent therapy with statin or placebo in the BELLES trial. ${ }^{2}$ Although LDL decreased, CAC progression did not improve in the treatment group. One thousand healthy men and women were treated with vitamin $\mathrm{C}$, alpha-tocopherol (vitamin $\mathrm{E}$ ), and a statin or with placebos in the St. Francis Heart Study. ${ }^{3}$ All participants had very high CAC scores. CAC progression was confirmed, and it was the same in both groups.

Intensive statin therapy has not been able to decrease CAC progression when compared to standard statin treatments. ${ }^{4}$ Neither therapy improved CAC progression. When chronic inflammation is detected, the liver produces more cholesterol in an attempt to reverse the inflammatory process. The ideal therapy should be avoidance of the offending etiological factors, not artificially decreasing cholesterol with statins.

Vitamin K2 deposits calcium in the skeletal and dental systems and prevents calcium from being deposited in arteries or other abnormal locations. A vitamin K2 deficiency causes CAC. Most people in modern societies are vitamin $\mathrm{K} 2$ deficient. No clinical trials are available comparing vitamin $\mathrm{K} 2 \mathrm{MK}-4$ vs. MK-7 for CAD. Until this information is known, it seems reasonable to include both vitamins $\mathrm{K} 2$ in a CAC reversal protocol because vitamin $\mathrm{K} 2$ has no known side effects. Both vitamin K2 are liposoluble and must be taken with fat. Trafarx.com sells a relatively inexpensive MK-4 in a powder form.

Up to $80 \%$ of the population suffers from a magnesium deficiency because, for many decades, farmers have not replaced magnesium in the soil. About 350 enzymes need magnesium to work properly, 
including energy producing reactions. Excess calcium/magnesium ratio intake increases $\mathrm{CAC}$. Based on current knowledge, any CAC reversing protocol should include: (A) avoiding inflammatory foods that cause $\mathrm{CAD}$; (B) replacing nutritional deficiencies, like vitamin $\mathrm{K} 2$, magnesium, and vitamin $\mathrm{D}$; and $(\mathrm{C})$ eliminating toxins that contribute to $\mathrm{CAD}$, like lead and other heavy metals. The whole health or functional protocol used in this case successfully decreased CAC.

\section{Conclusion}

A 12-month functional, whole health, protocol successfully lowered CAC without the need for any statins or other prescription medications.

\section{Acknowledgments}

None.

\section{Conflicts of interest}

None. Author has no pertinent financial interests with the subject matter or materials discussed.

\section{Funding}

None.

\section{References}

1. Joshi PH, Patel B, Blaha MJ , et al. Coronary artery Calcium predicts Cardiovascular events in participants with a low lifetime risk of Cardiovascular disease: The Multi-Ethnic Study of Atherosclerosis (MESA). Atherosclerosis. 2016;246:367-373.

2. Raggi P, Davidson M, Callister TQ, et al. Aggressive versus moderate lipid-lowering therapy in hypercholesterolemic postmenopausal women: Beyond Endorsed Lipid Lowering with EBT Scanning (BELLES). Circulation. 2005;112(4):563-571.

3. Arad Y, Spadaro LA, Roth M, et al. Treatment of asymptomatic adults with elevated coronary calcium scores with atorvastatin, vitamin $\mathrm{C}$, and vitamin E: the St. Francis Heart Study randomized clinical trial. J Am Coll Cardiol. 2005;46(1):166-172.

4. Schmermund A, Achenbach S, Budde T, et al. Effect of intensive versus standard lipid-lowering treatment with atorvastatin on the progression of calcified coronary atherosclerosis over 12 months: a multicenter, randomized, double-blind trial. Circulation. 2006;113(3):427-437. 